\title{
Presque tout sur CED-4, un chaperon pro-apoptogène du ver à l'homme
}

En mai 1997, médecine/sciences avait relaté la série de travaux permettant, enfin, d'avoir une idée de la fonction de la molécule CED-4 de Caenorhabditis elegans dans l'apoptose $\left(\mathrm{m} / \mathrm{s} n^{\circ} 5\right.$, vol. 13, p. 738). Trois gènes jouent un rôle essentiel dans l'apoptose du ver C. elegans: ced-9, dont l'homologue chez les vertébrés est $\mathrm{Bcl}$-2; ced-3, qui est l'archétype des caspases (cystéinyl-aspartyl protéases); et ced-4, qui n'avait jusqu'à présent pas d'homologue connu chez les vertébrés. Cependant, les travaux relatés en mai dans médecine/sciences indiquaient que la molécule CED-4 pouvait se fixer à la fois à CED-9 et à CED-3. Dans des extraits cellulaires de mammifères, en l'absence de CED-4, Bcl-X $\mathrm{L}_{\mathrm{L}}$ peut également être co-précipitée avec des caspases. Plusieurs expériences indiquaient que CED-4, synthétisée par des cellules de mammifères après transfection à l'aide d'un vecteur d'expression, se localisait à la membrane mitochondriale lorsque Bcl-2 était normale. En présence de mutants de Bcl-2 incapables de se fixer aux mitochondries, CED-4 était en position cytoplasmique. Enfin, il était possible d'isoler par immunoprécipitation de cellules transfectées à l'aide du vecteur d'expression de CED-4, un complexe $\mathrm{Bcl}_{\mathrm{L}} / \mathrm{CED}-$ 4/caspase. L'interprétation de tous ces résultats était que la localisation à la membrane mitochondriale du complexe Bcl-2 ou Bcl-X $/$ /CED-4 (ou son homologue de mammifère)/caspase inhibe l'apoptose alors que la libération de CED-4 dans le cytoplasme entraîne l'activation des caspases et l'apoptose. Des résultats récents, également rapportés dans médecine/sciences $\left(\mathrm{m} / \mathrm{s} \mathrm{n}^{\circ}\right.$ 5, vol. 13, déroulement de l'apoptose, les anomalies de perméabilité membranaire des mitochondries s'accompagnent précocement de la libération de cytochrome c qui semble capable de concourir à l'apoptose en activant les caspases. Restaient cependant plusieurs questions non résolues: (1) existe-t-il réellement un homologue de CED-4 chez les mammifères? (2) quel est exactement l'activité de CED-4 et de cet homologue éventuel sur l'activation des caspases? (3) existe-t-il une coordination entre la libération supposée de CED-4 de la membrane mitochondriale lors du déclenchement de l'apoptose et les autres phénomènes mitochondriaux décrits, notamment la libération de cytochrome c? Deux articles viennent de faire considérablement progresser les connaissances sur la nature et le rôle de CED-4 et de son homologue. L'article le plus important est manifestement celui de Zou et al. qui ont réussi à isoler et à caractériser l'homologue humain de CED4 [1]. Ces auteurs avaient développé un système biochimique acellulaire permettant d'étudier l'activation de la caspase 3. Cela leur permit d'isoler un complexe protéique indispensable à la maturation protéolytique de cette caspase, associée à son activation. Trois protéines au moins semblent faire partie de ce complexe, dénommées Apaf (apoptosis proteaseactivating factors). Apaf-2 est le cytochrome c et Apaf-1 est une protéine d'un poids moléculaire de 130000 , présentant de nettes analogies de structure et de séquence avec CED-4. La partie amino-terminale de CED-4 aussi bien que d'Apaf-1 comportent un domaine CARD (caspase-recruitment domain), leur permettant très probablement d'interagir avec les caspases. Dans Apaf-1, mais non dans CED-4, se trouve un domaine de type WD-40, connu pour participer à des interactions interprotéiques. On ne connaît pas le rôle de ce domaine, mais les auteurs suggèrent qu'il pourrait permettre l'interaction avec Apaf-2/cytochrome c, et peut-être avec Apaf-3. Chinnaiyan et al., dans une correspondance scientifique à Nature [2] précisent le mode d'action de CED-4. Ces auteurs montrent que CED-4 possède un site de fixation de l'ATP, ayant potentiellement une activité ATPasique. L'ATP est indispensable pour que CED-4 catalyse l'activation de la caspase de $C$. elegans, CED-3. CED-4 et le complexe des Apaf semblent donc catalyser l'activation d'une ou de plusieurs caspases qui, en cascade, entraînent alors l'activation des autres molécules de cette famille. Cependant, rien n'indique dans la séquence de CED4/Apaf-1 que ces molécules aient par elles-mêmes une activité de protéase. Plus probablement, on peut regarder CED-4/Apaf-1 comme une protéine chaperon, capable de conférer aux caspases auxquelles elles sont liées, en présence d'ATP, une conformation propice à leur auto-activation protéolytique. Pour intégrer les données disponibles, il faut encore faire l'hypothèse que cette activité de CED-4/Apaf serait inhibée lorsque ce complexe est lié à des molécules de type $\mathrm{Bcl}-2 / \mathrm{Bcl}_{\mathrm{L}}$ fixées à la membrane mitochondriale. Peut-être peut-on alors voir le phénomène d'activation de l'apoptose de la manière suivante: une modification de la perméabilité de la membrane mitochondriale, modulée par le jeu de formation de canaux aux proprié- 
tés différentes impliquant des molécules anti-apoptotiques (Bcl-2, Bcl$\mathrm{X}_{\mathrm{L}}$ ) ou pro-apoptotiques (Bax, Bak) [3], entraîne la dissociation du complexe CED-4/Apaf-1-caspase des molécules de type $\mathrm{Bcl}-2 / \mathrm{Bcl}_{\mathrm{L}}$ liées à la membrane mitochondriale, et la formation d'un nouveau complexe impliquant Apaf-2/cytochrome c. Peut-on imaginer que le cytochrome c libéré par les mitochondries entre en compétition avec la liaison de CED-4/Apaf-1 aux molécules Bcl-2/ $\mathrm{Bcl}_{\mathrm{L}}$ ? Quoi qu'il en soit, Apaf2 /cytochrome c coopérerait avec CED-4/Apaf-1 pour stimuler, en présence d'ATP, l'auto-activation protéolytique d'une ou plusieurs caspases et la poursuite de la cascade apoptotique.

A.K.
1. Zou H, Henzel WJ, Liu X, Lutschg A, Wang $\mathrm{X}$. Apaf-1, a human protein homologous to $C$. elegans CED-4, participates in cytochrome cdependent activation of caspase-3. Cell 1997; $90: 405-13$.

2. Chinnaiyan AM, Chaudhary D, O'Rourke K, Koorin EV, Dixit VM. Role of CED-4 in the activation of CED-3. Nature 1997 ; 388 : 728-9.

3. Antonsson B, Conti F, Ciavatta AM, Montessuit $\mathrm{S}$, Lewis $\mathrm{S}$, et al. Inhibition of Bax channelforming activity of Bcl-2. Science 1997; 277 : 370-2.

\section{BRÈVES}

Mécanismes de la spécificité du facteur pro-apoptotique TRAIL pour les cellules cancéreuses. Cela fait environ deux ans qu'a été découverte la molécule TRAIL, une protéine susceptible de déclencher l'apoptose de très nombreuses cellules normales de la rate, du poumon, de la prostate, etc. Le facteur TRAIL et son récepteur DR4 (death receptor 4) sont, en effet, synthétisés par de très nombreuses cellules, normales ou malignes. Cependant, paradoxalement, beaucoup de cellules cancéreuses sont résistantes à l'apoptose induite par TRAIL, contrairement aux cellules non transformées correspondantes. Afin de comprendre les bases de cette particularité, aux évidentes implications thérapeutiques, trois équipes se sont efforcées de mettre en évidence de nouveaux récepteurs de TRAIL qui pourraient expliquer cet effet différentiel sur les cellules normales et transformées. Deux de ces équipes publient leurs résultats dans les numéros du
8 août de la revue Science $[1,2]$ alors que la troisième, également américaine, s'apprête à les publier dans J. Exp. Med. [3]. Un autre récepteur, DR5, possédant un death domain (partagé par les récepteurs pro-apoptotiques de la famille Fas/TNFR) a été ainsi identifié. En fait, il se comporte un peu comme DR4 et est capable de relayer un signal apoptotique. En revanche, la recherche de molécules ayant le domaine de fixation de TRAIL et dépourvu de death domain a permis de mettre en évidence une molécule désignée par le sigle TRID (trail receptor without an intracellular domain) par Pan et al. [1] et DcRl (decoy receptor) par Sheridan et al. [2]. Naturellement, en l'absence de death domain, ce récepteur est incapable de transmettre un signal apoptotique mais reste fonctionnel en ce qui concerne la liaison de TRAIL. De ce fait, l'hyperexpresssion de ce récepteur tronqué protège des cellules normales sinon sensibles à l'action pro-apoptotique de TRAIL, probablement en entrant en compétition avec DR4 et DR5 pour la liaison de TRAIL. Chose très intéressante, expliquant probablement la résistance des cellules cancéreuses à TRAIL, ces dernières sont dépourvues de TRID/DcRl qui est exprimé à la membrane des cellules normales, lié par une ancre glycosyl-phosphatidylinositol (protéine glypiée). Naturellement, ces résultats incitent les chercheurs concernés, notamment la Société Genentech de San Francisco (CA, USA), à tester l'efficacité de TRAIL in vivo visà-vis de tumeurs greffées. Les premiers résultats seraient que TRAIL n'a, en effet, pas de toxicité générale et on attend avec anxiété des informations sur son éventuel effet antitumoral.

[1. Pan G, et al. Science 1997; 277 : 815-8.]

[2. Sheridan JP, et al. Science 1997; 277: 818-21.]

[3. Gura T. Science 1997; 277 : 768.] 